\title{
DIGITAL PROCESSES IN PRODUCT CREATION
} An Extended Abstract

\author{
Dr. Gustav J. Olling
}

DaimlerChrysler Corp., 800 Chrysler Drive, Auburn Hills, MI 48326, USA

gjo@daimlerchrysler.com

\begin{abstract}
This paper underlines the importance of focusing on organization, culture, technology, and education to evolve an expanding global enterprise in today's complex and dynamic business climate.
\end{abstract}

Key words: product and component teams; cultural changes; improvement-oriented; CAD technology; well-integrated databases; information bridges; integrate expertise; global engineers; universities; conclusion.

\section{INTRODUCTION}

In an era of corporate expansion into global markets and mergers with international partners, many companies are discovering weaknesses in their organizational structures. Personnel, process, and technical solutions that worked well on a smaller scale do not necessarily succeed on a world stage. Which organizational strategies will support rapid product development and adaptation to market shifts while maintaining consistent productivity and quality levels?

In effecting large-scale organizational change, it is important to avoid overemphasizing the role of technology. Heavy investment in technology for technology's sake in the 1980s and 1990s demonstrated that changing an enterprise's technical framework without altering the ingrained attitudes and practices of the enterprise does not produce the expected gains in efficiency. Successful enterprise innovation results from broad organizational and cultural changes (80\%) supported by suitable technological changes $(20 \%)$.

Please use the following format when citing this chapter:

Olling, Gustav, J., 2006, in International Federation for Information Processing (IFIP), Volume 207, Knowledge Enterprise: Intelligent Strategies In Product Design, Manufacturing, and Management, eds. K. Wang, Kovacs G., Wozny M., Fang M., (Boston: Springer), pp. 21-25. 


\section{ORGANIZATION}

One enterprise strategy that can be used to improve a company's infrastructure is to organize product development personnel into product and component teams. An automobile company may have previously partitioned its work into component groups, such as powertrain, chassis, body, and electrical, that worked on all vehicle types and operated separately from other groups, such as manufacturing, procurement, marketing, and finance. Under the new scheme, product teams divide the work by product type (Small Vehicle, Premium Vehicle, Family Vehicle, Activity Vehicle, Truck, and Powertrain), while the component teams continue to apply expertise and innovation to major components of the vehicle. The product and component teams work together on each new vehicle program. Moreover, these crossfunctional teams include representatives from manufacturing, procurement, marketing, and finance. Hence, the collective effort becomes more collaborative and focused. Communication among groups improves considerably, and the closer cooperation enables flexibility and the ability to change direction quickly.

\section{CULTURE}

Organizational changes must be accompanied by a complementary set of cultural changes if the benefits of the structural changes are to be fully realized. For example, a manufacturing enterprise can establish a Product Development System to guide the product development process through a series of quality gates. It can launch a number of process improvement programs to analyze and enhance various manual, technical, and managerial activities, and it can establish Centers of Competence to encourage the exchange and capture of good ideas for key product areas. The net effects of these changes are twofold. First, they establish clear guidelines for how product development work is to be done, and, second, they provide procedures for doing the work and forums for discussing how to improve the work processes. Over time, these changes help to replace traditional resultsoriented, "don't blame me" thinking with improvement-oriented, "let's get better" thinking. The cultural change releases an abundance of energy and fosters a climate of open communication. 


\section{TECHNOLOGY}

While organizational and cultural changes are being made, technological changes continue to occur in the areas of computer-aided engineering, manufacturing automation, and management information systems. Many global enterprises today are trying to integrate the product development and manufacturing processes from end to end as much as possible. The consolidated product teams described above, insofar as they are integrative in nature, help to synchronize the activities of product development, process development, and resource planning. These efforts are supported by the evolution of design technology from manual and electronic drafting to CAD modeling to feature-based design to knowledge-based engineering.

Feature-based design possesses the virtue of storing rules, relationships, and attributes of the product-in-development in such as way as to support end-to-end integration efforts. Although knowledge-based engineering is still evolving, it will enable the automation of product and process design activities, associativity between the requirements of these activities, the simultaneous conduct of the activities, and eventually a seamless development cycle for product and process.

While intelligent CAD systems are essential for a growing global enterprise, they are not the only key technological element. On the back end, the enterprise needs well-integrated databases. The strategy in this area is to gather the sources of product geometry, bills of material, change notice information, engineering and manufacturing standards, resource capacity, long-range plans, and a myriad of other information types into an integrated data system. In a large international company that must function at full capacity every day, this is a daunting task. It comes as no surprise to say that most large enterprises have not achieved such corporate-wide integration.

Most companies can, however, make two important strides. They can adopt and customize a Product Data Management system to organize geometry, specify product configurations, and control changes. In addition, they can create in-house web applications that act as integration points between disparate databases. These measures allow companies to reference and exchange vital product information while evolving a more integrated set of databases.

Another important advancement that global enterprises can implement is the construction of information bridges, such as a Company Portal, that facilitates communication between corporate groups in different countries and between the company and its suppliers. The improved communication afforded by these bridges promotes part sharing and reuse, closer cooperation, and coordinated planning. 
Current research is providing additional tools to assist expanding global organizations. The emergence of more intelligent process models and supporting systems, the evolution of decision-support, expert, and optimization systems, and the design of logical models that analyze the relationship of events and suggest alternative actions - all these will integrate expertise into the support structure of the enterprise. The thinking behind product creation will be increasingly automated.

\section{EDUCATION}

Besides the three factors so far discussed - organization, culture, and technology - one additional element is crucial to the viability of the expanding global enterprise: Education. The complex and changing environment of today's global businesses requires an extended set of job skills. Global engineers must be not only technical specialists but also product strategists, operation integrators, and ethical practitioners. They must solve unstructured, multi-solutional, and mutable problems. Unfortunately, educational institutions do not produce an abundance of people who have mastered the creative process and can communicate to all levels of an organization. Even fewer graduates appreciate the virtues of craftsmanship. To encourage global thinking, universities must expose students to a wide range of technical and liberal arts subjects, a wide range of computer-aided design, engineering, manufacturing, and customerfeedback processes, and a wide range of exercises in how to communicate with coworkers, management, and customers.

In addition to improving curricula, universities can hire faculty with broad educational backgrounds and diversified practical experience. They can support the efforts of faculty members to update their research skills and technical expertise. They can build laboratories, or work with independent laboratories, that realistically model current industrial practice, simulate variable manufacturing systems, and support quantitative analysis of processes and results. Finally, universities can collaborate with commercial enterprises to exchange ideas and methods pertaining to the latest technology.

\section{CONCLUSION}

To remain competitive, the expanding global corporation must make sure that it has the infrastructure to deal effectively with the speed and complexity of current industrial changes. The enterprise must be organized to focus on well-defined product areas with a comprehensive team of 
planners, developers, and implementers. It must establish a corporate culture with well-defined rules for all aspects of the product creation lifecycle, a cooperative climate of process improvement, and a community spirit that promotes the free exchange of ideas. The enterprise must continue to strive for improved integration of information and the automation of expert knowledge and decision-making. Finally, the enterprise must look for and encourage the education of creative, adaptive, honest, resilient individuals to deal with the difficult global issues of today and tomorrow. 